ANNALES

POLONICI MATHEMATICI

$81.1(2003)$

\title{
Remarks on the generalized index of an analytic improper intersection
}

\author{
by Krzysztof Jan NowaK (Kraków)
}

\begin{abstract}
This article continues the investigation of the analytic intersection algorithm from the perspective of deformation to the normal cone, carried out in the previous papers of the author $[7,8,9]$. The main theorem asserts that, given an analytic set $V$ and a linear subspace $S$, every collection of hyperplanes, admissible with respect to an algebraic bicone $B$, realizes the generalized intersection index of $V$ and $S$. This result is important because the conditions for a collection of hyperplanes to be admissible with respect to $B$ are of geometric nature: it is not necessary to analyse the embedded components of the intersections involved, but only the supports of the intersections of $B$ with successive hyperplanes.
\end{abstract}

1. Introduction. The generalized index at a point $P$ of improper intersection of a purely dimensional analytic set $V$ with a submanifold $S$ was defined by Tworzewski [10] as the extended multiplicity at $P$ of the total result of the analytic intersection algorithm performed for a generic admissible collection of smooth divisors. It is, of course, possible to confine oneself to the case where $S$ is a linear subspace (flattening of $S$ ).

In the papers $[8,9]$ we reduced the computation of the generalized index at a point $P$ of improper intersection of a purely dimensional analytic set $V$ with a submanifold $S^{s}$ of a complex manifold $M^{m}$ to the study of intersections of an algebraic bicone $B$ with linear hyperplanes. We proved that this index is realized by a collection of smooth hypersurfaces provided that their equations satisfy certain generic conditions of filter-regularity. Those conditions are of linear character: the first derivatives of the equation of each successive hypersurface should avoid a finite union of proper linear subspaces (depending on the previous divisors of the collection). In particular,

2000 Mathematics Subject Classification: 14C17, 32S10, 32C25, 32B10.

Key words and phrases: analytic improper intersections, analytic intersection algorithm, generalized intersection index, intersection multiplicity, deformation to the normal cone, algebraic bicones.

Research partially supported by KBN grant No. 5 P03A 00521. 
the generalized intersection index of $V$ and a linear subspace $S$ is realized by a generic collection $\mathcal{H}=\left(H_{1}, \ldots, H_{r}\right)$ of hyperplanes.

The purpose of this article is to show that the generalized intersection index of $V$ and a linear subspace $S$ is realized by every collection of hyperplanes admissible with respect to the foregoing algebraic bicone $B$ (main theorem). This fact is important because the conditions for a collection of hyperplanes to be admissible with respect to $B$ are of geometric nature: it is not necessary to analyse the embedded components of the intersections involved (in other words, it is not necessary to analyse the embedded primary components of the ideals obtained in the analytic intersection algorithm). The choice of a collection of hyperplanes which realizes the generalized intersection index depends only on the support of the algebraic bicone $B$; successive hyperplanes of such a good collection can be picked by taking into consideration only the supports of the intersections with the previous hyperplanes. The main theorem of the paper applies both to theoretic research and concrete calculations. In particular, it can be used to express the generalized intersection index of two analytic curves in terms of their local parametrizations (cf. [6, Sect. 4] and Sect. 5 of this article).

2. Analytic intersection algorithm. We recall the analytic intersection algorithm, which is a local analogue of the Stückrad-Vogel intersection algorithm from global algebraic geometry (cf. $[10,7,9]$ ). For an open subset $U$ of the complex manifold $M^{m}$ such that $U \cap S^{s} \neq \emptyset$, denote by $\mathcal{H}(U)$ the set of all $\mathcal{H}=\left(H_{1}, \ldots, H_{r}\right)$, where $r:=m-s$ is the codimension of $S$ in $M$, satisfying the two conditions:

1) $H_{k}(k=1, \ldots, r)$ is a smooth hypersurface in $U$ containing $S \cap U$;

2) $\bigcap_{k=1}^{r} T_{P} H_{k}=T_{P} S$ for each point $P$ in $S \cap U$ (here $T_{P} N$ is the tangent space to a manifold $N$ at a point $P$ ).

For a given analytic subset $V$ in $M$ of pure dimension $d$, let $\mathcal{H}(U, V)$ be the set of all $\mathcal{H}=\left(H_{1}, \ldots, H_{r}\right) \in \mathcal{H}(U)$ such that $((U \backslash S) \cap V) \cap H_{1} \cap \ldots \cap H_{k}$ is an analytic subset in $U \backslash S$ of pure dimension $d-k$ (or is empty) for $k=1, \ldots, r$. We call such an $\mathcal{H}$ a collection of smooth divisors admissible with respect to $V$.

For each $\mathcal{H}=\left(H_{1}, \ldots, H_{r}\right) \in \mathcal{H}(U, V)$, the analytic intersection algorithm consists in constructing two families of analytic cycles on $U$ by means of the recursive formula:

$$
\alpha_{-1}=[V \cap U], \quad H_{0}:=U,
$$

and next

$$
\varrho_{k}=\left(\alpha_{k-1} \cdot H_{k}\right)^{S}, \quad \alpha_{k}=\alpha_{k-1} \cdot H_{k}-\varrho_{k} ;
$$

here $Z^{S}$ denotes the part of an analytic cycle $Z$ supported by $S$. By def- 
inition, the above intersection products are proper. Consequently, $\alpha_{k}$ is a $(d-k)$-cycle on $U$ and $\varrho_{k}$ is a $(d-k)$-cycle on $S \cap U ; \varrho_{k}$ will be called the result of the kth step of the intersection algorithm carried out for the collection $\mathcal{H}$. The total result of the intersection algorithm is the analytic cycle

$$
\varrho=\varrho(V \cdot \mathcal{H}):=\sum_{k=0}^{r} \varrho_{k}
$$

on the submanifold $S$.

For an analytic cycle $Z=\sum_{i=0}^{m} Z_{i}$ on a complex manifold $M$, where $Z_{i}$ is an $i$-cycle $(i=0, \ldots, m)$, the extended multiplicity of $Z$ at a point $P$ is the sequence

$$
\text { ext.mult }_{P} Z:=\left(\operatorname{mult}_{P} Z_{m}, \ldots, \operatorname{mult}_{P} Z_{0}\right) \in \mathbb{N}^{m+1} \text {. }
$$

For any point $P$ in $S$, Tworzewski [10] defined the multi-index

$$
\widetilde{g}(V, S)(P):=\min \left\{\operatorname{ext} \operatorname{mult}_{P} \varrho(V \cdot \mathcal{H}): \mathcal{H} \in \mathcal{H}(U, V), P \in U\right\} \in \mathbb{N}^{s+1},
$$

where both neighbourhoods $U$ and admissible collections $\mathcal{H}(U, V)$ vary, and the minimum is with respect to the lexicographic ordering. The $(s+1)$-tuple $\widetilde{g}(V, S)(P)$ is called the generalized (or extended) intersection index of $V$ and the submanifold $S$ at the point $P$; the sum $g(P)$ of the components of $\widetilde{g}(P)$ is called the index (or multiplicity) of intersection of $V$ with $S$ at $P$.

3. Deformation to an algebraic bicone $B$. The generalized intersection index is realized for generic collections of hypersurfaces. In $[8,9]$ we provided sufficient conditions for such a good collection, namely, certain linear conditions on the tangent spaces at $P$ to the hypersurfaces of the collection. The generalized intersection index of $V$ and $S$ at $P$ coincides with the bidegree sequence of an algebraic bicone $B$, and the intersection multiplicity is the multiplicity at $P$ of the normal cone $C:=C_{V \cap S} V$ (see $[7,8,9]$ and also [1,2]). Those formulae can be established by the method of deformation to the normal cone, applied to the analytic intersection algorithm. We sketch this method briefly.

One may, of course, assume that the ambient space is $\mathbb{C}^{m}=\mathbb{C}_{u}^{s} \times \mathbb{C}_{v}^{r}$, $S=\left\{(u, v) \in \mathbb{C}_{u}^{s} \times \mathbb{C}_{v}^{r}: v=0\right\}$, and that $P=0$. In order to deform the analytic set $V$ to the normal cone $C:=C_{V \cap S} V$ (cf. [7, 9]), define the following analytic family of analytic sets:

$$
\bigcup_{t} V_{t}=\left\{(u, v ; 1: t) \in \mathbb{C}^{m} \times \mathbb{P}_{1}:(u, v / t) \in V\right\}
$$

parametrized by $t \in \mathbb{C} \backslash\{0\}$; the deformation space $\mathcal{V}$ for $V$ is the closure of the above family in $\mathbb{C}^{m} \times \mathbb{P}_{1}$. The normal cone $C=C_{V \cap S} V$ to the subspace $V \cap\{v=0\}=V \cap S$ in $V$ (the intersection is understood in the ideal-theoretic 
sense) is the fibre $V_{\infty}$ over the point $\infty \in \mathbb{P}_{1}$; the normal cone $C$ is thus an analytic space, not necessarily reduced. Observe that the spaces $V_{t} \cap S$ do not vary and all coincide with the zero-section (vertex space) $V \cap S$ of the normal cone. Moreover, all the fibres $V_{t}$ for $t \in \mathbb{C}, t \neq 0$, are isomorphic to $V$.

One can treat $C=V_{\infty}$ as an analytic cycle, determined by the fibre. Therefore, by additivity, also analytic cycles can be deformed, and this deformation procedure commutes with taking cycles (see e.g. [9, Chapt. II, Sect. 3]). From now on we shall regard normal cones as analytic cycles. The normal cone $C=V_{\infty}$ is the limit cycle near $P$ for the family of analytic cycles $V_{t}$ when $t$ approaches $\infty$; we then say that $C$ is the limit cycle for $V$.

Now we recall deformation to an algebraic bicone (cf. [8, 9]), which plays an important role in the investigation of the analytic intersection algorithm. One can repeat the foregoing construction of deformation, but with the coordinates $u$ and $v$ interchanged. In other words, we now deform the cone $C$ to the normal cone $B$ to the fibre of $C$ over the point $P$ of the vertex space. It is easy to see that $B$ coincides with the tangent cone to $C$ at $P$, and thus $B$ is an algebraic cone, which is also an algebraic bicone with respect to the variables $u$ and $v$. The bicone $B=C_{\infty}$ is again the limit cycle near $P$ for the family of analytic cycles $C_{t}$ when $t$ approaches $\infty$; we then say that $B$ is the limit cycle for $C$.

4. Main theorem. If $B$ is an algebraic bicone, any collection of hyperplanes $\mathcal{H}$ admissible with respect to $B$ realizes (by the classical Bézout theorem) the generalized intersection index of $B$ and $S$; the choice of such a collection depends only on the support of the cycle $B$. We shall prove that any such $\mathcal{H}$ is admissible and realizes the generalized indices at $P$ for the intersection of $C$ with $S$ and of $V$ with $S$.

Main Theorem. Let $V$ be an analytic set of pure dimension $d$ at the point $P=0 \in \mathbb{C}^{m}$ and $S$ be a linear subspace of $\mathbb{C}^{m}$. We may, of course, assume that $\mathbb{C}^{m}=\mathbb{C}_{u}^{s} \times \mathbb{C}_{v}^{r}(m=s+r)$ and $S=\left\{(u, v) \in \mathbb{C}_{u}^{s} \times \mathbb{C}_{v}^{r}: v=0\right\}$. Consider the normal cone $C:=C_{V \cap S} V$ and an algebraic bicone $B$ that is the tangent cone to $C$ at $P$; both $C$ and $B$ are regarded as analytic cycles (cf. $[7,8,9])$. Suppose a collection of hyperplanes $\mathcal{H}=\left(H_{1}, \ldots, H_{r}\right)$ is admissible with respect to $B$. Then $\mathcal{H}$ is admissible and realizes the generalized indices at $P$ for the intersection of $C$ with $S$ and of $V$ with $S$.

Proof. According to the theorem on deformation to the normal cone (cf. $[7,9]$ ) and the theorem on deformation to an algebraic bicone (cf. $[8,9]$ ), we have the equalities

$$
\widetilde{g}(V, S)(P)=\widetilde{g}(C, S)(P)=\widetilde{g}(B, S)(P)=:\left(g_{0}, g_{1}, \ldots, g_{r}\right)
$$


and all the above generalized intersection indices coincide with the bidegree sequence of the algebraic bicone $B$. Denote by $\bar{\alpha}_{k}$ and $\bar{\varrho}_{k}$ the two families of analytic cycles constructed by means of the analytic intersection algorithm performed for the bicone $B$ and the collection $\mathcal{H}=\left(H_{1}, \ldots, H_{r}\right)$ of hyperplanes.

We assert that the collection $\mathcal{H}$ is admissible for both $C$ and $V$, and thus it is possible - as before when carrying out the intersection algorithm-to construct analytic cycles $\alpha_{k}^{*}, \varrho_{k}^{*}$ and $\alpha_{k}, \varrho_{k}(k=0,1, \ldots, r)$. We shall prove this assertion by induction on $k$. We shall simultaneously show that $\bar{\alpha}_{k}$ are the limit cycles for $\alpha_{k}^{*}$ (when deforming to the algebraic bicone $B$ ), $\alpha_{k}^{*}$ are the limit cycles for $\alpha_{k}$ (when deforming to the normal cone $C$ ), and that

$$
\operatorname{mult}_{P} \varrho_{k}=\operatorname{mult}_{P} \varrho_{k}^{*}=\operatorname{mult}_{P} \bar{\varrho}_{k}=g_{k} \quad(k=0,1, \ldots, r) .
$$

We shall consider only the case of deformation to the algebraic bicone $B$, since the case of deformation to the normal cone $C$ is analogous. Observe that every hyperplane $H_{k}$ of $\mathcal{H}$ is of the form

$$
H_{k}=\left\{(u, v) \in \mathbb{C}_{u}^{s} \times \mathbb{C}_{v}^{r}: \sum_{i=1}^{r} c_{k i} v_{i}=0\right\}
$$

and is invariant under both the deformations. Clearly, $\bar{\alpha}_{0}:=B-B^{S}$ is the limit cycle for $\alpha_{0}^{*}:=C-C^{S}$ (cf. $\left.[8,9]\right)$. Hence

$$
\text { mult }_{P} \varrho_{0}^{*} \leq \text { mult }_{P} \bar{\varrho}_{0}=g_{0} \leq \operatorname{mult}_{P} \varrho_{0}^{*} .
$$

Further, by continuity of proper intersections of positive analytic cycles (cf. $[4,11])$, the hyperplane $H_{1}$ meets properly the cycles $C_{t}-C_{t}^{S}$ for $t \neq 0$, and

$$
\bar{\alpha}_{0} \cdot H_{1}=\bar{\varrho}_{1}+\bar{\alpha}_{1} \quad \text { with } \quad \bar{\varrho}_{1}:=\left(\bar{\alpha}_{0} \cdot H_{1}\right)^{S}
$$

is the limit cycle for

$$
\alpha_{0}^{*} \cdot H_{1}=\varrho_{1}^{*}+\alpha_{1}^{*} \quad \text { with } \quad \varrho_{1}^{*}:=\left(\alpha_{0}^{*} \cdot H_{1}\right)^{S} .
$$

Then we get

$$
\operatorname{mult}_{P} \varrho_{1}^{*} \leq \operatorname{mult}_{P} \bar{\varrho}_{1}=g_{1} \leq \operatorname{mult}_{P} \varrho_{1}^{*}
$$

whence

$$
\operatorname{mult}_{P} \varrho_{1}^{*}=\operatorname{mult}_{P} \bar{\varrho}_{1}=g_{1},
$$

and thus $\bar{\alpha}_{1}$ is the limit cycle for $\alpha_{1}^{*}$. Repeating the above argument for the intersection with $\mathrm{H}_{2}$, we obtain the equality

$$
\operatorname{mult}_{P} \varrho_{2}^{*}=\operatorname{mult}_{P} \bar{\varrho}_{2}=g_{2},
$$

and consequently $\bar{\alpha}_{2}$ is the limit cycle for $\alpha_{2}^{*}$. In this manner we can continue the analytic intersection algorithm, and thereby verify the assertion for the analytic cone $C$ (a routine induction argument).

Summing up, $\mathcal{H}$ proves to be a collection of hyperplanes admissible for $C$, and it realizes the generalized intersection index of $C$ and $S$ at $P$. An 
analogous reasoning for the deformation to the analytic cone $C$ shows that $\mathcal{H}$ is an admissible collection for $V$, and it also realizes the generalized intersection index of $V$ and $S$ at $P$. This completes the proof of our theorem.

5. Applications. One can apply the main theorem of the paper to provide new short proofs of two known effective formulae for the generalized intersection index of two analytic curves $V_{1}$ and $V_{2}$ in terms of their local parametrizations. This was done in the joint paper [6] (Sect. 4, Theorem 2) of T. Krasiński and the author, by means of a description of the normal cone

$$
C:=C_{\left(V_{1} \times V_{2}\right) \cap \Delta}\left(V_{1} \times V_{2}\right),
$$

where $\Delta$ is the diagonal, via local parametrization of the curves. Whereas the first formula - due to Chądzyński, Krasiński, Tworzewski [3] — refers to an isolated intersection, the second one - due to Krasiński [5] - is concerned with self-intersection. We now recall those formulae:

Consider two irreducible curve germs $V_{1}, V_{2}$ at $0 \in \mathbb{C}^{m}$, having a common tangent line, parametrized as follows:

$$
\left(x_{1}=t^{p}, x_{k}=\varphi_{k}(t)\right) \quad \text { and } \quad\left(x_{1}=t^{q}, x_{k}=\psi_{k}(t)\right), \quad k=2,3, \ldots, m,
$$

with $\operatorname{ord}_{0} \varphi_{k}>p$ and $\operatorname{ord}_{0} \psi_{k}>q$. Then the generalized intersection index $\widetilde{g}=\left(g_{0}, g_{1}, g_{2}\right)$ of $V_{1}$ and $V_{2}$ at 0 is equal to

$$
\widetilde{g}= \begin{cases}\left(0,0, \frac{1}{q} \sum_{i=1}^{q} \operatorname{ord}_{0}\left(\varphi\left(t^{q}\right)-\psi\left(\varepsilon^{i} t^{p}\right)\right)\right) & \text { if } V_{1} \neq V_{2}, \\ \left(0, p, \sum_{i=1}^{p-1} \operatorname{ord}_{0}\left(\varphi(t)-\varphi\left(\varepsilon^{i} t\right)\right)\right) & \text { if } V_{1}=V_{2}=V,\end{cases}
$$

where $\varepsilon$ is a primitive root of unity of degree $q$ (in the second case $p=q$ and $\varphi(t)=\psi(t))$.

We conclude the paper with an observation which follows immediately from the main theorem.

The set of those good collections of hyperplanes

$$
\mathcal{H}=\left(H_{1}, \ldots, H_{r}\right) \in \mathcal{H}(U, V)
$$

which realize the generalized intersection index of $V$ and $S$, contains a dense Zariski-open subset of the set of all collections of hyperplanes containing $S$.

Yet the problem to fully characterize the set of good collections of hyperplanes remains open. 


\section{References}

[1] R. Achilles and M. Manaresi, Multiplicities of a bigraded ring and intersection theory, Math. Ann. 309 (1997), 573-591.

[2] R. Achilles and S. Rams, Intersection numbers, Segre numbers and generalized Samuel multiplicities, Arch. Math. (Basel) 77 (2001), 391-398.

[3] J. Chądzyński, T. Krasiński and P. Tworzewski, On the intersection multiplicity of analytic curves in $\mathbb{C}^{n}$, Bull. Polish Acad. Sci. Math. 45 (1997), 163-169.

[4] E. M. Chirka, Complex Analytic Sets, Kluwer, 1989.

[5] T. Krasiński, Improper intersection of analytic curves, Univ. Iagel. Acta Math. 39 (2001), 153-166.

[6] T. Krasiński and K. J. Nowak, Intersections of analytic curves, Ann. Polon. Math. 80 (2003), 193-202.

[7] K. J. Nowak, Analytic improper intersections I: deformation to the normal cone, Bull. Polish Acad. Sci. Math. 48 (2000), 121-130.

[8] -, Analytic improper intersections II: deformation to an algebraic bicone and applications, ibid., 131-140.

[9] -, Improper intersections in complex analytic geometry, Dissertationes Math. 391 (2001).

[10] P. Tworzewski, Intersection theory in complex analytic geometry, Ann. Polon. Math. 62 (1995), 177-191.

[11] P. Tworzewski and T. Winiarski, Continuity of intersection of analytic sets, ibid. 42 (1983), 387-393.

Institute of Mathematics

Jagiellonian University

Reymonta 4, 30-059 Kraków, Poland

E-mail: nowak@im.uj.edu.pl

Reçu par la Rédaction le 15.1.2002

Révisé le 16.5.2002 